\title{
Localization-delocalization transition of a polaron near an impurity
}

\author{
A. S. Mishchenko, ${ }^{1,2}$ N. Nagaosa, ${ }^{1,3}$ A. Alvermann, ${ }^{4}$ H. Fehske,${ }^{4}$ G. De Filippis,${ }^{5}$ V. Cataudella, ${ }^{5}$ and O. P. Sushkov ${ }^{6}$ \\ ${ }^{1}$ Cross-Correlated Materials Research Group (CMRG), ASI, RIKEN, Wako 351-0198, Japan \\ ${ }^{2} R R C$ "Kurchatov Institute", 123182, Moscow, Russia \\ ${ }^{3}$ Department of Applied Physics, The University of Tokyo, 7-3-1 Hongo, Bunkyo-ku, Tokyo 113, Japan \\ ${ }^{4}$ Institut für Physik, Ernst-Moritz-Arndt-Universität Greifswald, 17489 Greifswald, Germany \\ ${ }^{5}$ Coherentia-CNR-INFM and Dip. di Scienze Fisiche - Università di Napoli Federico II - I-80126 Napoli, Italy \\ ${ }^{6}$ School of Physics, University of New South Wales, Sydney 2052, Australia
}

\begin{abstract}
We solve the problem of polaron localization on an attractive impurity by means of direct-space Diagrammatic Monte Carlo implemented for the system in the thermodynamic limit. In particular we determine the ground state phase diagram in dependence on the electron-phonon coupling and impurity potential strength for the whole phonon frequency range. Including the quantum phonon dynamics we find and characterize a new phase which is missing in the zero phonon-frequency limit (adiabatic approximation), where self-trapped polarons are not localized at shallow impurities. We predict and show that in the vicinity of the localization transition a region with a mixture of weakand strong-coupling spectral response is realized.
\end{abstract}

PACS numbers: 71.10.Fd, 71.38.-k, 02.70.Ss

A general approach to the theoretical description of a particle in a bulk medium coupled both to bosonic excitations and the potential of imperfections is an important but notoriously hard problem that poses a real challenge even to modern nonperturbative approaches [1]. As yet only approximate results, relying, e.g., on dynamicalmean field theory exist. A central question in this context is the formation of three-dimensional (3D) polarons at impurities, or the Anderson localization of polarons in disordered media [2, 3, 4]. The overall importance of the physics of electron-phonon interaction in doped materials makes this issue of general interest for different areas of physics and technology. As a matter of fact the interplay between disorder and interaction effects is an important issue for contemporary materials design. For example high temperature superconductors [5, 6, 7] or materials with colossal magnetoresistance [8] are doped Mott insulators where besides the coupling to bosonic excitations (phonons and magnons) disorder is present.

In this Letter we present the exact solution to the polaron problem in the presence of an attractive impurity in a $3 \mathrm{D}$ material. The accepted model for that situation is given by the Hamiltonian $H=H^{(0)}+H^{(1)}$ with

$$
\begin{aligned}
& H^{(0)}=-U c_{\mathbf{0}}^{\dagger} c_{\mathbf{0}}+\omega_{\mathrm{ph}} \sum_{\mathbf{i}} b_{\mathbf{i}}^{\dagger} b_{\mathbf{i}}, \\
& H^{(1)}=-t \sum_{\langle\mathbf{i}, \mathbf{j}\rangle} c_{\mathbf{i}}^{\dagger} c_{\mathbf{j}}-\gamma \sum_{i}\left(b_{\mathbf{i}}^{\dagger}+b_{\mathbf{i}}\right) c_{\mathbf{i}}^{\dagger} c_{\mathbf{i}} .
\end{aligned}
$$

In $H^{(0)}, U$ is the attractive impurity potential for the electron $c_{0}^{\dagger}$ at site 0 and $b_{i}^{\dagger}$ creates a dispersionless optical phonon with frequency $\omega_{\text {ph }}$ at Wannier $i . H^{(1)}$ describes the electron transfer $\propto t$ between nearest neighbor sites and local Holstein coupling to the phonons $\propto \gamma$.

In the absence of electron-phonon (el-ph) coupling $(\gamma=0)$, the critical $U$ for particle localization at the impurity is $U_{c}(\gamma=0) \approx 3.96[9]$; all energies are measured in units of $t$ hereafter. In the adiabatic approximation (AA), setting $\omega_{\mathrm{ph}}=0$, the phase diagram in $U-\lambda$ coordinates, with the dimensionless coupling constant $\lambda=\gamma^{2} /\left(6 t \omega_{\mathrm{ph}}\right)$, was established in Ref. [10]. The phase boundary in AA, separating delocalized polaron states from localized ones, crosses the $U$-axis at $U_{c}(\gamma=0)$ and the $\lambda$-axis at $\lambda_{c}\left(U_{c}=0, \omega_{\mathrm{ph}}=0\right) \approx 0.9$. The latter crossing is a confusing property of the AA phase diagram since it implies that for el-ph couplings $\lambda>\lambda_{c}\left(U_{c}=0, \omega_{\mathrm{ph}}=0\right)$ the polaron is localized even when $U=0$. Quite the contrary, a particle is never localized in a translationally invariant lattice $(U=0)$ with quantum phonons $\left(\omega_{\mathrm{ph}}>0\right)$. Instead the particle undergoes only a crossover from the weak-coupling light polaron to a strong-coupling heavy polaron with small radius around a self-trapping coupling $\lambda_{\mathrm{ST}}$ [11]. The AA erroneously equates $\lambda_{\mathrm{ST}}$ with the critical el-ph coupling strength $\lambda_{c}$ required for polaron localization at $U \neq 0$. Therefore drastic differences between the exact result and that obtained in AA are expected, especially at small $U_{c}$.

Having this delicate situation in mind, we decided to study the full Hamiltonian (12)-(2) with quantum phonons. To this end we employ a new scheme combining the Diagrammatic Monte Carlo (DMC) method in direct space [12] and the Stochastic Optimization Method for analytic continuation [12, 13] which provides the approximation-free solution of the above problem without finite size errors and in zero temperature limit. Calculating the charge density distribution (CDD) around the impurity and the local density of states (LDOS) on the impurity site we establish the exact localization phase diagram for different phonon frequencies. We characterize two novel polaronic regimes in a system with impurities. The polaron at small $U$ can be self-trapped though extended and not yet confined by shallow impurities. Another regime, arising near the critical parameters for lo- 
calization at the impurity, shows spectroscopic response like a mixture of spectra typical for weak, intermediate, and strong coupling.

The direct space DMC method [14] can provide the direct space Green functions (GFs) in imaginary time $(\tau)$ representation at zero temperature $G_{\mathbf{i j}}(\tau)=$ $\left\langle\operatorname{vac}\left|c_{\mathbf{j}}(\tau) c_{\mathbf{i}}^{\dagger}\right| \operatorname{vac}\right\rangle$ for the Hamiltonian (11-2) by Feynman diagram expansion in the interaction representation

$G_{\mathbf{i j}}(\tau)=\left\langle e^{-\tau H^{(0)}} \widehat{T}_{\tau}\left[\left\langle c_{\mathbf{j}}(\tau) c_{\mathbf{i}}^{\dagger} \exp \left\{-\int_{0}^{\tau} H^{(1)}\left(\tau^{\prime}\right) d \tau^{\prime}\right]\right\}\right\rangle\right.$.

The implementation of DMC 14 requires to keep in computer memory all GFs $\left\{G_{\mathbf{i j}}(\tau)\right\}$ in direct space, which restricts the lattice to about $25 \times 25$ sites. To avoid this size limitation we calculate only quantities related to onsite GFs $G_{\mathbf{i i}}(\tau)$. With our implementation of DMC we are able to calculate the on-site GFs at zero temperature for a $10^{8} \times 10^{8} \times 10^{8}$ lattice, thereby avoiding any finitesize or finite temperature errors. A slight modification of Eq. (3),

$n(\mathbf{i})=\left\langle\frac{e^{-\beta H^{(0)}}}{Z} \widehat{T}_{\tau}\left[\left\langle c_{\mathbf{i}}(\beta) c_{\mathbf{i}}^{\dagger} \exp \left\{-\int_{0}^{\beta} H^{(1)}\left(\tau^{\prime}\right) d \tau^{\prime}\right]\right\}\right\rangle\right.$,

introduces the estimator for the CDD at temperature $T=\beta^{-1}$. To make a calculation of the CDD feasible, we collect its statistics in a cube with $40^{3}$ number of sites. Note that this strategy does not introduce finite-size errors because only the $\tau=0, \beta$ points of the partition function loop are confined to the $40^{3}$-cube while the diagrams are free to sample all $\left(10^{8}\right)^{3}$ sites.

The CDD estimator is effective for locating the localization parameters for large $U$ only but, because of the requirement of finite temperatures, fails at small $U \ll t$. Note that the path-integral quantum Monte-Carlo algorithm [15], which is another method relevant for the problem formulated above [4], has serious precision limits for the same reason. Hence, the only rigorous method to locate the localization point in the infinite system is to calculate the on-site zero temperature GF $G_{\mathbf{i i}}(\tau)$, determine the LDOS $L_{\mathbf{i}}(\omega)=-\pi^{-1} \operatorname{Im} G_{\mathbf{i i}}(\omega)$ by analytic continuation [12, 13], and check for the presence of a bound state in the LDOS $L_{\mathbf{0}}(\omega)$ at the impurity site.

To validate the new implementation of the DMC technique, we located the critical $U_{c}(\lambda=0) \approx 3.96$ by calculating the CDD, normalized to unity at the impurity site, around the impurity. It occurred that for $U \leq U_{c}$ the charge density does not decrease exponentially with distance from the impurity while for $U>U_{c}$ it does. Perfect agreement is found between CDD obtained by DMC and that obtained in Ref. [9]. For $U$ close to $U_{c}$, however, determination of the LDOS $L_{\mathbf{0}}(\omega)$ is a much more precise method, since the CDD requires finite temperatures.

First let us demonstrate how trapped polaron states are determined using the LDOS. From the commutator
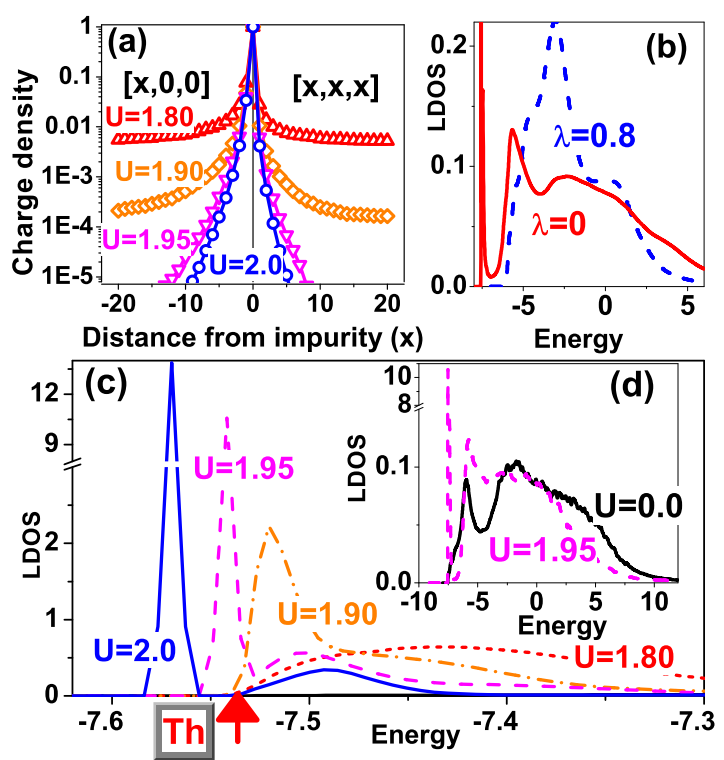

FIG. 1: (color online) Charge density at $T=0.001$ (a) and LDOS at the impurity site for $T=0(\mathrm{c}, \mathrm{d})$ for different values of $U$ at $\omega_{\mathrm{ph}}=2$ and $\lambda=0.8$. The arrow in panel (c) indicates the lower threshold (Th) of the spectrum at $\lambda=0.8$ and $U=0$. Panel (b) shows LDOS at the impurity site at $U=2$, $\omega_{\mathrm{ph}}=2$ for $\lambda=0$ (dashed line) and $\lambda=0.8$ (solid line). Statistical errorbars in panel (a) are less than $10^{-5}$ (i.e. much less than the point size).

$\left[H, c_{i}^{\dagger}\right]$ we find that, independent of the el-ph coupling $\lambda$, the first moment $M_{1}$ of the LDOS $L_{\mathbf{i}}(\omega)$ obeys

$$
M_{1}=\int_{-\infty}^{\infty} d \omega \omega L_{\mathbf{i}}(\omega)=-U \delta_{\mathbf{i}, \mathbf{0}}
$$

In accordance with the sum rule (5), the LDOS at the impurity site shifts to lower energies with increasing $U$ (Fig. 1r). The second moment $M_{2}=\int_{-\infty}^{\infty} d \omega \omega^{2} L_{\mathbf{i}}(\omega)$ increases with $\lambda$ (Fig. 1b) and the overall LDOS broadens. Figure 1 shows the CDD (a) and LDOS (c,d) for various values of $U$ at fixed $\lambda=0.8$ and $\omega_{\mathrm{ph}}=2$. Let us start the discussion with the case $U=0$, where no localization is expected. We determine the lower border $E_{\mathrm{Th}}(\lambda)$ of the LDOS $L_{0}(\omega)$ for given $\lambda$ (arrow in Fig. 1(c)). Increasing $U$ the LDOS changes but there is no spectral density below $E_{\operatorname{Th}}(\lambda)$ up to $U \leq 1.90$ (Fig. 1(c)). The CDD around the impurity, in accordance with the absence of a bound state in LDOS, does not show exponential decrease too (Fig. 1(a)). This gives another confirmation for the method employed here. In order to search for the localization-delocalization transition we proceed to larger values of $U$. For $U \geq 1.95$, the bound state appears below the threshold $E_{\mathrm{Th}}(\lambda)$ (Fig. 1(c)), and the CDD decays exponentially (Fig. 1(a)). In this way we obtain one transition point in the phase diagram (Fig. 2(a)), here $U_{c}\left(\omega_{\mathrm{ph}}=2, \lambda=0.8\right)=1.925 \pm 0.025$. Recall that, although the LDOS approach needs a very precise deter- 
mination (compare (Fig. 1(c) and (d)), it is applicable for any values of $\lambda$ and $U$. On the contrary, the CDD method is fast but, due to requirement of finite temperature, not reliable at $U \ll 1$.
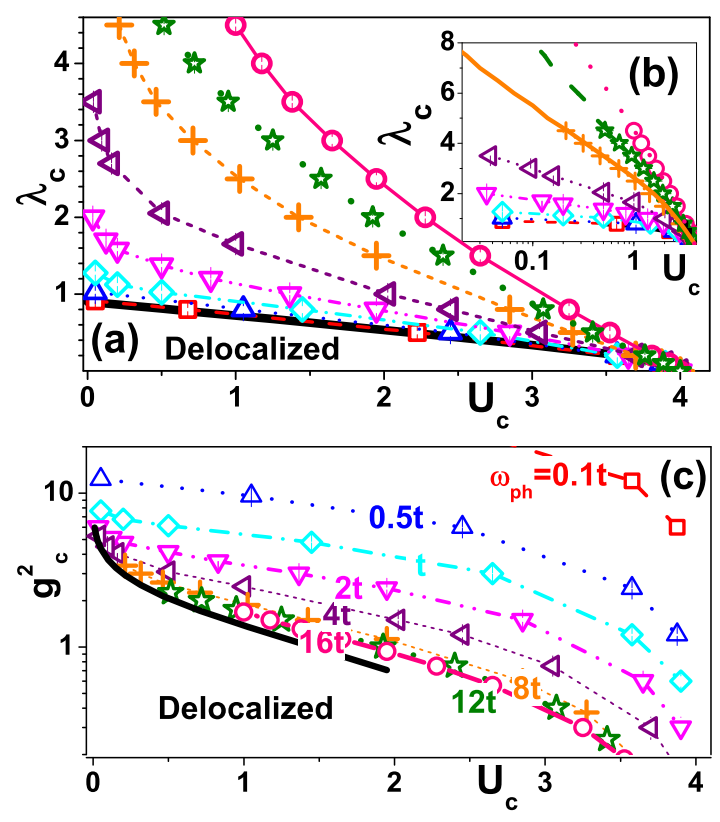

FIG. 2: (color online) Phase boundaries between delocalized (left lower corner) and localized states of a polaron in $\lambda_{c}-$ $U_{c}(\mathrm{a}, \mathrm{b})$ and $g_{c}^{2}-U_{c}(\mathrm{c})$ coordinates: adiabatic limit $\omega_{\mathrm{ph}}=$ 0 (thick solid line in panel (a)), $\omega_{\text {ph }}=0.1$ (squares), 0.5 (triangles up), 1 (diamonds), 2 (triangles down), 4 (triangles left), 8 (crosses), 12 (stars), 16 (circles). The thick solid curve in panel (c) is obtained from Eq. (6). Solid, dashed and dotted lines in panel (b) are results obtained by the CBS method [16] for $\omega_{\mathrm{ph}}=8,12$, and 16 . The values of $\lambda_{c}$ are set exactly while the errorbars of the quantity $U_{c}$ are less than $3.0 \times 10^{-2}$.

Next the phase diagram for polaron localization is presented in Fig. 2] Using $\lambda_{c}-U_{c}$ coordinates (Fig. 2(a), (b)), we see how our exact solution differs from the adiabatic result ( $\omega_{\mathrm{ph}} \rightarrow 0$, thick solid curve) for finite $\omega_{\mathrm{ph}}$. In Fig. 2(c), with $g^{2}-U_{c}$ coordinates $\left(g=\gamma / \omega_{\mathrm{ph}}\right)$, we show the deviation from the limiting phase boundary at $\omega_{\mathrm{ph}} \rightarrow \infty$ (thick solid curve),

$$
U_{c}\left(\omega_{\mathrm{ph}}=\infty\right)=U_{c}(\gamma=0) \exp \left(-g_{c}^{2}\right) .
$$

This relation is obtained by Lang-Firsov transformation, which renormalizes hopping, and accordingly the critical $U_{c}$, as $t \rightarrow t \exp \left(-g^{2}\right)$. Note that a exponential relation between $U_{c}$ and $g_{c}^{2}$ (or $\lambda_{c}=g_{c}^{2} \omega_{\mathrm{ph}} / 6 t$ ) is a characteristic property of the small $U_{c} \ll 1$ and large $\lambda>1$ regime (Fig. 2(b)), since for large $\omega_{\mathrm{ph}}$,

$$
\lambda_{c}\left(U_{c}, \omega_{\mathrm{ph}}\right) \simeq\left(\omega_{\mathrm{ph}} / 6 t\right) \ln \left[U_{c}(\gamma=0) / U_{c}\right]+\text { const } .
$$

It is indeed seen in Fig. 2(b) that the slope of the phase boundary increases with $\omega_{\mathrm{ph}}$.

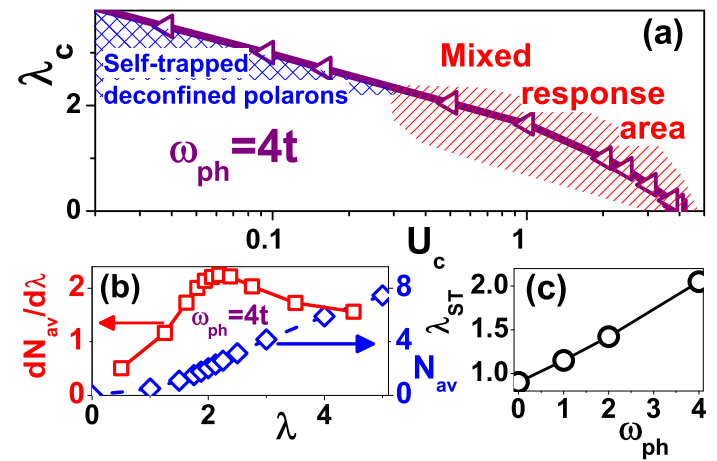

FIG. 3: (color online) (a) Localization phase diagram and (b) average number of phonons $N_{\mathrm{av}}$ and the derivative $d N_{\mathrm{av}} / d \lambda$ for $\omega_{\mathrm{ph}}=4$. (c) Dependence of the self-trapping coupling $\lambda_{\mathrm{ST}}$ on the phonon frequency $\omega_{\mathrm{ph}}$. Errorbars are less than point size.

We emphasize the excellent agreement of the novel DMC approach with the adiabatic limit [10] and antiadiabatic limit Eq. (6) results, as well as with the data obtained by the Coherent Basis States (CBS) method [16] for small $U$, which proves the validity of the implementation. Note that the phase diagram obtained here is free from any substantial error and presents the first available solution of the Hamiltonian Eq. (11)-(2) for all parameter regimes.

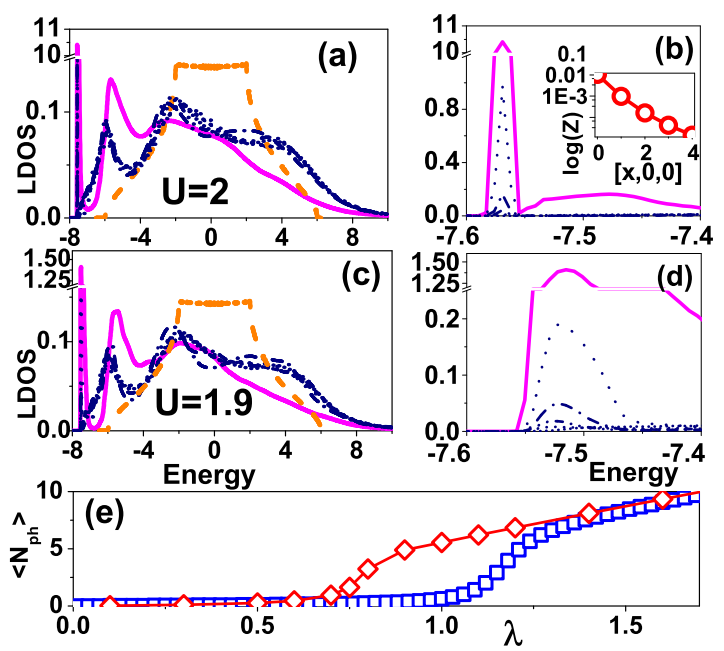

FIG. 4: (color online) LDOS for a localized polaron $(\mathrm{a}, \mathrm{b})$ at $U=2$ and a delocalized polaron $(\mathrm{c}, \mathrm{d})$ at $U=1.9$, where $\lambda=0.8$ and $\omega_{\mathrm{ph}}=2$. Shown is the LDOS at the impurity site $(0,0,0)$ (solid line), nearest neighbor $(1,0,0)$ (dots), site $(2,0,0)$ (dash-dots), $(3,0,0)$ (dash-dot-dots), $(4,0,0)$ (shortdashes), and for infinite distance from the impurity (short dots). The dashed line is the unperturbed $\operatorname{LDOS}(\lambda=0$, $U=0$ ). The inset in panel (b) gives the $Z$-factor of the LDOS $\delta$-peak of the bound state as a function of the distance to the impurity. Panel (e) shows the average number of phonons at (diamonds) and far from the impurity (squares) for $\omega_{\mathrm{ph}}=1$ and $U=1.4$. 
Let us finally discuss two essential features of the phase diagram, which are entirely missing in the adiabatic approximation. The first is realized at large el-ph couplings where the polarons are already self-trapped but not yet confined by shallow impurities (cross-hatched region in Fig. 3(a)). The self-trapping coupling $\lambda_{\mathrm{ST}}$, locating the crossover from the weak- to strong-coupling regime, can be defined, e.g., as the maximum of the derivative of the average number of phonons in the ground state $N_{\mathrm{av}}=\left\langle b_{\mathbf{q}=\mathbf{0}}^{\dagger} b_{\mathbf{q}=\mathbf{0}}\right\rangle$ with respect to the coupling constant $\lambda$ (Fig. 3(b,c)). For small enough $U \ll 1$, and any given $\omega_{\mathrm{ph}}$, one finds a sector in the phase diagram (Fig. 3(a)) where $\lambda_{\mathrm{ST}}\left(\omega_{\mathrm{ph}}\right)<\lambda<\lambda_{c}\left(U, \omega_{\mathrm{ph}}\right)$. This sector defines the phase of self-trapped deconfined polarons, whose identification is obtained here for the first time.

The second novel feature appears at moderate values of $U$ close to the transition region between localized and extended states (line-shaded area in Fig. 3a). There, the spectral properties of a polaron are strongly positiondependent. In Fig. 4 we show the LDOS calculated at, and in the vicinity of the impurity. Both for a localized $(\mathrm{a}, \mathrm{b})$ and extended $(\mathrm{c}, \mathrm{d})$ polaron the LDOS at the impurity site strongly differs from that at the nearest neighbor site in the low-energy region $(b, d)$. On the contrary, the overall features of LDOS at the nearest neighbor are very similar to those at infinite distance from the defect (Fig. 㤢,c). This property points out how strongly the spectral properties depend on the value of the impurity potential at a given lattice site. Comparison of the average number of phonons at the impurity with that in infinite distance to the impurity (Fig. 44) shows that for a wide range of parameters the lattice is weakly distorted far from the impurity while it is strongly deformed near the impurity. This demonstrates how impurities enhance the formation of small polarons.

As a consequence it is expected that in a material with imperfections a mixture of behavior typical for weak-coupling polarons (far away from an impurity) and strong-coupling polarons (close to, or at, the impurity) occurs. Even though the impurity concentration can be small, the induced changes, e.g. in the spectral response, can be drastic. For example, for $U=1.90$ and $\lambda=0.8$ (Fig. [a) the charge density on the impurity site is four orders of magnitude larger than in the bulk of the system. Therefore, even a small impurity concentration $n_{i} \approx 10^{-3}$ suffices to entirely change the spectral properties. For example, since photoemission [17] and optical conductivity [18] spectra are a very different for weak and strong-coupling, one can expect very rich mixture of the spectral responses.

In conclusion, introducing the direct space diagrammatic Monte Carlo in the thermodynamic limit we presented the exact phase diagram for localization of a polaron at an attractive impurity for all coupling strengths, values of the impurity potential, and phonon frequencies ranging from the adiabatic to the antiadiabatic regime.
Most notably we characterize a novel phase where heavy polarons are mobile in the presence of shallow impurities and predict complex spectral properties of the systems close to the localization-delocalization transition. The present DMC method can be easily generalized to study more general situations, e.g. systems with longrange particle hopping, impurities with long-range attractive/repulsive potentials, or interfaces and layered structures, demonstrating the potential for future research.

A.S.M. is supported by RFBR 07-02-00067a. N.N. was partly supported by the Grant-in-Aids from under the Grant No. 15104006, No. 16076205, No. 17105002, No. 19048015, and NAREGI Nanoscience Project from the Ministry of Education, Culture, Sports, Science, and Technology, Japan. A.A. and H.F. acknowledge support by DFG SFB 652 .

[1] O. Gunnarsson, M. Calandra, and J. E. Han, Rev. Mod. Phys. 75, 1085 (2003).

[2] S. M. Girvin and M. Jonson, Phys. Rev. B 22, 3583 (1980); M. H. Cohen, E. N. Economou, and C. M. Soukoulis, Phys. Rev. Lett. 51, 1202 (1983).

[3] F. X. Bronold, A. Alvermann and H. Fehske, Phil. Mag 84, 673 (2004); A. Alvermann and H. Fehske, Phys. Rev. B 77, 045125 (2008).

[4] J. P. Hague, P. E. Kornilovitch and A. S. Alexandrov, arXiv: 0805.2556 .

[5] P. A. Lee, N. Nagaosa and X.-G. Wen, Rev. Mod. Phys. 78, 17 (2006).

[6] O. Gunnarsson and O. Rösch, J. Phys.: Condens. Matter 20043201 (2008).

[7] V. Cataudella et al, Phys. Rev. Lett. 99, 226402 (2007); A. S. Mishchenko et al, ibid 100, 166401 (2008).

[8] A. J. Millis, Phys. Rev. B 53, 8434 (1996); V. Perebeinos and P. B. Allen, Phys. Rev. Lett. 85, 5178 (2000)

[9] G. F. Koster and J. C. Slater, Phys. Rev. 96, 1208 (1954).

[10] Y. Shinozuka and Y. Toyozawa, J. Phys. Soc. Jpn. 46, 505 (1979).

[11] A. S. Mishchenko et al, Phys. Rev. B 66, 020301 (2002); A. Alvermann, H. Fehske and S. A. Trugman, arXiv: 0803.3425.

[12] A. S. Mishchenko et al, Phys. Rev. B 62, 6317 (2000); A. S. Mishchenko, Phys. Usp. 48, 887 (2005). A. S. Mishchenko and N. Nagaosa, J. Phys. Soc. J. 75, 011003 (2006);

[13] K. Vafayi and O. Gunnarsson, Phys. Rev. B 76, 035115 (2007).

[14] A. Macridin, G.A. Sawatzky, and M. Jarrell, Phys. Rev. B 69, 245111 (2004).

[15] P. E. Kornilovitch, Phys. Rev. Lett. 81, 5382 (1998).

[16] V. Cataudella et al., Phys. Rev. B 70, 193105 (2004); G. De Filippis et al., ibid. 72, 014307 (2005).

[17] A. S. Mishchenko, N. Nagaosa, Phys. Rev. Lett. 93, 036402 (2004)

[18] A. S. Mishchenko et al, Phys. Rev. Lett. 91, 236401 (2003); G. De Filippis et al, ibid. 96, 136405 (2006). 